\title{
CASO -2016: Sindrome de Churg Strauss: a propósito de dos casos en el Hospital San Vicente de Paúl
}

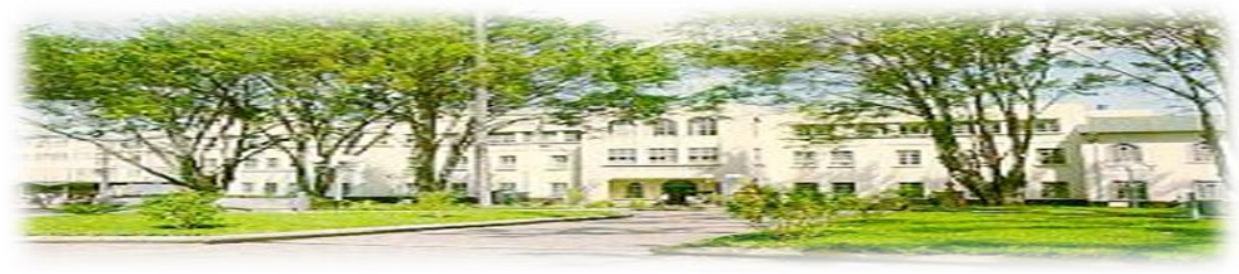

Hospital San Juan de Dios, San José, Costa Rica. Fundado en 1845

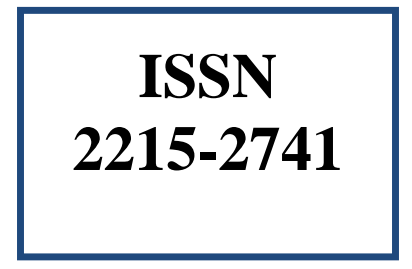

$\begin{array}{ll}\text { Recibido: } & 24 / 02 / 2014 \\ \text { Aceptado: } & 06 / 10 / 2016\end{array}$

Huixian Hu Liang 1

Andrés Leitón Chaves 2

Claudio Salas Mena 3

\begin{abstract}
${ }^{1}$ Medicina General y Cirugía, Universidad Latina de Costa Rica. hhu2986@gmail.com
2 Medicina General y Cirugía, Universidad Autónoma de Centro América. Profesor universitario de la cátedra de medicina en Universidad Autónoma de Centro América. andres131c@@hotmail.com

3 Medicina General y Cirugía, UCR. Médico especialista en Reumatología, UCR. Profesor Universitario UCIMED. Profesor de Internado Universitario Hospital San Vicente de Paul.
\end{abstract}

\section{RESUMEN}

Las vasculitis asociadas a anticuerpos citoplasmáticos antineutrófilos, clasificadas como vasculitis de pequeños vasos incluyen: granulomatosis con poliangeítis (enfermedad de Wegener), poliangeítis microscópica y la granulomatosis eosinofilica con poliangeítis (GEPA) (Churg Strauss). La enfermedad de Churg Strauss es una de las vasculitis necrotizantes sistémicas más raras, pero potencialmente mortal. La incidencia internacional de la enfermedad es de aproximadamente 2.5 casos por cada 100 000 adultos por año, el rango de supervivencia a 1 año es de $90 \%$ y a los 5 años es de $62 \%$. El síndrome de Churg Strauss (SCS) tiene una fisiopatología compleja, en la cual contribuyen factores genéticos y agentes extrínsecos; también, diferentes tipos de células, como células T, eosinófilos, células B y células residentes participantes en la reacción inflamatoria y la lesión tisular. Se han descrito tres fases de evolución de la enfermedad parcialmente superpuestas. Una primera fase prodrómica caracterizada por manifestaciones atópicas como asma, rinitis alérgica, sinusitis y pólipos nasales. La segunda fase se caracteriza por eosinofilia periférica, y pueden ocurrir infiltrados eosinofílicos en pulmones y tracto gastrointestinal. Durante la tercera fase de la enfermedad ocurren manifestaciones vasculíticas. Además de los síntomas constitucionales también hay manifestaciones osteomusculares como mialgias, artralgias y artritis. El pronóstico del SCS ha mejorado dramáticamente desde la introducción de los corticoesteroides, y estos siguen siendo la primera línea de terapia de la GEPA. Aunque la piedra angular del tratamiento son los 
corticoesteroides, la enfermedad severa es tratada de manera similar a otras vasculitis asociadas a ANCA, con otros agentes inmunosupresores como ciclofosfamida. Sin embargo el manejo sigue siendo un reto, con estudios clínicos limitados.

\section{PALABRAS CLAVE}

Granulomatosis, vasculitis, poliangeitis, ANCA, miocardiopatía, corticosteroides, eotaxinas.

\section{ABSTRACT}

Associated vasculitis to antineutrophil cytoplasmatic antibodies, clasified as small vessel vasculitis that includes: polyangiitis whit granulomatosis ( wegener disease) microscopic polyangiitis and eosinophilic granulomatosis with polyangiitis ( Churg Strauss). Churg Strauss disease is one of the rarest systemic necrotizing vasculitis, but potenctially deadly. International incidieses for this disease is 2.5 cases per every 100.000 adults per year, the range of survival to 1 year is $90 \%$ and to 5 years is $62 \%$. The Churg Strauss syndrome has a complex fisiopathology, in which genetic factors and external agentes are involved: also different types of cells, like T cells, eosinophils, B cells, and inflammatory cells. There are 3 phases on the evolution of the disease. The first phase is characterized for atopic demostrations like asthma, allergic rhinitis and nasal polyps. The second phase is characterized for periferic eosinophilia and eosinophilic infiltrates in lungs and gastrointestinal tract. During the third phase of the disease there are vasculitic demonstrations. Besides constitutional symptons there is also musculokeletal demonstrational like myalgia, arthralgia and arthritis. The Churg Strauss syndrome prognosis has improve drastically since the introduccion of corticosteroids therapy and it's the first line of therapy. Even do the cornerstone of treatment are the corticosteroids, the severe presentation of this disease is treated in similar way as any other vasculitis associated with ANCA, whit other inmunossuppresive drugs like cyclophosphamide, however treatment is still a challenge, with limited clinical studies.

\section{KEY WORDS}

Granulomatosis, vasculitis, polyangiitis, ANCA, cardiomyopathy, corticosteroids, eotaxins.

\section{CASOS CLÍNICOS}

\section{Caso Clínico \#1}

Paciente masculino de 65 años, residente de Heredia, extabaquista, acude al servicio de emergencias del Hospital San Vicente de Paul, por historia de dolor en miembros inferiores de intensidad 7/10 de más de 1 mes de evolución, dolor agravado por el movimiento, asociado a cansancio general impidiéndole así una marcha adecuada. Como antecedentes patológicos de importancia se destaca asma con un diagnóstico desde hace 37 años tratada con Becotide, Oxis, Salbutamol y Montelukast.

Al examen físico inicial el paciente presenta una frecuencia cardiaca en 112 latidos por minuto, presión arterial en $104 / 61 \mathrm{mmHg}$ y temperatura corporal dentro de rangos normales, el examen físico general es normal y se realiza finalmente un test de fuerza muscular MMT8 con un valor de 65 puntos (flexores 10, deltoides 10, bíceps 9, extensión de antebrazo 8 , glúteo mayor 7 , glúteo menor 7 , cuádriceps 7, dorsiflexión del pie 7).

\section{nico:}

Estudios complementarios del caso clí-

En el estudio de sangre se reporta una hemoglobina en $14.1 \mathrm{~g} / \mathrm{dL}$ con un hematocrito de $42 \%$, leucocitos en $68.43 \times 10^{\wedge} 3 / \mathrm{uL}$ y una hipereosinófilia de $77.5 \%$ $\left(47.9 \times 10^{\wedge} 3 / \mathrm{uL}\right)$. Una velocidad de eritrosedimentación en $102 \mathrm{~mm} / \mathrm{H}$ y proteína $\mathrm{C}$ reactiva $>120 \mathrm{mg} / \mathrm{L}$. El estudio de velocidad de conducción nerviosa reporta: estudio compatible con polineuropatía motora y sensitiva de tipo desmielinizante secundariamente axonal. El TAC de tórax muestra leves opacidades intersticiales y en vidrio esmerilado bilaterales, engrosamiento broncovascular y opacidades centrolubulillares parcialmente definidas.

La biopsia pulmonar reporta además un tejido pulmonar con infiltrado inflamatorio intersticial e intraalveolar con evidencia de abundantes eosinófilos. Se realiza un TAC de senos paranasales con hallazgos compatibles con sinusitis crónica asociado a polipósis nasal. Se toma una muestra de músculo de miembro inferior izquierdo para biopsia reportando un tejido muscular esquelético que presenta a nivel de las zonas de tejido conectivo in- 
termuscular y a nivel vascular un infiltrado inflamatorio compuesto por células plasmáticas y eosinofilos que involucran la pared de algunos vasos sanguíneos de pequeños y mediano calibre generando una vasculitis necrotizante. ANCA C $(4.2 \mathrm{U} / \mathrm{ml})$ negativo y ANCA $\mathrm{P}$ positivo $(>100 \mathrm{U} / \mathrm{ml})$.

\section{Análisis del caso:}

De acuerdo a la historia clínica del paciente, el examen físico y las abundantes y variadas pruebas complementarias realizadas, se piensa finalmente en el diagnóstico de síndrome de Churg Strauss, cumpliendo este caso con los 6 criterios del American College of Rheumatology (1990) para la clasificación de esta patología, en los cuales se requieren 4 criterios para su diagnóstico. El paciente se egresa para continuar su manejo de forma ambulatoria, con control en Reumatología y el Centro Nacional de Rehabilitación. Se revisará la información más actualizada de esta enfermedad a propósito del caso.

\section{Caso clínico \#2}

Paciente masculino de 54 años, residente de Heredia, acude al Hospital San Vicente de Paul, por historia de dificultad en la marcha asociada a disminución de la sensibilidad en miembros inferiores, aquejando además artralgias de más de un mes de evolución. Como antecedentes de importancia, el paciente es conocido asmático y con historia de sinusitis crónica, en tratamiento con beconase, beclometasona inhalada y prednisona.

Al examen físico presenta una presión arterial de 119/84mm/Hg, saturación de oxígeno en $93 \%$ con una frecuencia cardiaca en $931 \mathrm{pm}$. Al momento de la consulta presenta un buen estado general con su examen físico dentro de los límites normales, excepto por una fuerza muscular disminuida en miembros inferiores y pérdida de sensibilidad, es notable una atrofia bilateral de músculos gastrocnemios.

Estudios complementarios del caso clínico:

En el estudio de sangre se reporta: Hemoglobina: $12.1 \mathrm{~g} / \mathrm{dl}$, hematocrito: 36\%, Leucocitosis: 43.6X10^3/uL, Eosinófilos en $60 \%$, velocidad de eritrosedimentación: $40 \mathrm{~mm} / \mathrm{h}$ y una proteína $\mathrm{C}$ reactiva en $13 \mathrm{mg} / \mathrm{dl}$, examen general de orina normal, pruebas de función hepática y renal normales,
NT-PROBNP: 225pg/ml, ANCAc: 3.50, ANCAp: 27.77. Se realizan además los siguientes estudios: una broncoscopía que reporta un árbol bronquial endoscópicamente normal, un ecocardiograma con resultado normal, biopsia de nervio sural izquierdo: normal.

Electromiografía: diagnostico compatible con poli neuropatía motora y sensitiva de tipo axonal y desmielinizante severa. Biopsia de pulmón: parénquima pulmonar que presenta edema y fibrina, asociados a un infiltrado inflamatorio mixto con presencia de neutrófilos y eosinófilos, a nivel de los tabiques alveolares, se observa vasos sanguíneos sin evidencia sugestiva de vasculitis. No hay evidencia de granulomas.

El TAC senos paranasales reportó presencia de engrosamiento mucoso difuso que compromete todos los senos paranasales, de aspecto polipoideo en senos maxilares de hasta $10 \mathrm{~mm}$ de diámetro, complejos osteomeatales permeables (hallazgos compatibles con sinusitis crónica difusa, hallazgos sugestivos de poliposis maxilar bilateral). TAC DE TORAX: derrame pleural bilateral asociado a leve atelectasia compresiva bilateral, pequeños focos bilaterales de consolidación peribronquial.

\section{Análisis del caso:}

En el caso de este paciente tenemos muchos de los hallazgos encontrados en el caso \#1, la biopsia tomada, en este caso de nervio sural, tuvo un resultado normal. Se piensa igualmente en el diagnóstico de Síndrome de Churg Strauss por su historia clínica y la gran cantidad de estudios que apoyan este diagnóstico. Se revisará la información más actualizada de esta enfermedad a propósito de los casos presentados.

\section{DISCUSIÓN Y ANÁLISIS DEL CASO}

\section{Definición}

Las vasculitis asociadas a anticuerpos citoplasmáticos antineutrófilos, clasificadas como vasculitis de pequeños vasos incluyen: granulomatosis con poliangeítis (enfermedad de Wegener), poliangeítis microscópica y la granulomatosis eosinofilica con poliangeítis (GEPA) (Churg Strauss). Aunque comparten muchas características la GEPA, tiene ciertas especificidades como asma, eosinofilia san- 
guínea y tisular y afectación frecuente de oídos, nariz y garganta. La enfermedad de Churg Strauss es una de las vasculitis necrotizantes sistémicas más raras, pero potencialmente mortal, con afectación predominante de pequeños vasos. En su evolución natural la enfermedad cuenta con 3 fases: la asmática o prodrómica, la eosinofílica y la vasculítica. Cada fase cuenta con una variación en la presentación clínica del cuadro, lo cual dificulta su diagnóstico. ${ }^{1,2,3}$

\section{Epidemiología}

La incidencia del síndrome del Churg Strauss en Estados Unidos es 1-3 casos por cada 100000 adultos por año. La incidencia internacional es de aproximadamente 2.5 casos por cada 100000 adultos por año.

Raza: En algunos estudios no se ha sugerido ninguna predilección por Churg Strauss en ninguna raza, en otros, sin clara documentación se ha sugerido que la enfermedad de Churg Strauss comparte con otras vasculitis sistémicas una tendencia a presentar una mayor prevalencia en individuos blancos de ascendencia Nórdica. Sin embargo se requiere de información adicional para confirmarlo.

$\underline{\text { Sexo }}$ : así como en un gran número de otras vasculitis sistémicas primarias, los hombres son levemente más susceptibles para desarrollar la enfermedad de Churg Strauss, el rango es de 1.4:1.

$\underline{E d a d:}$ la mayoría de las personas con la enfermedad de Churg Strauss experimentan la aparición de la enfermedad en el rango de 15-69 años. El pico de aparición de las manifestaciones de vasculitis es en la media de la cuarta década de la vida, en las otras vasculitis sistémicas primarias, la edad de aparición es más tardía, entre 60-65 años.

La enfermedad de Churg Strauss es principalmente una enfermedad del adulto, ocurriendo en individuos que desarrollan asma en la adultez. Las etapas de vasculitis de la enfermedad de Churg Strauss rara vez, o nunca, se manifiesta en niños pequeños, aunque pueden aparecer en la adolescencia. La enfermedad de Churg Strauss de inicio en la niñez es menos probable de complicarse con vasculitis del $\mathrm{SNC}$ que los casos que se desarrollan en individuos de edad media.

Mortalidad/ morbilidad: las principales causas de morbilidad y mortalidad en el síndrome de Churg
Strauss son miocarditis e infarto del miocardio, secundarios a arteritis coronaria. Con tratamiento, el rango de supervivencia a 1 año es de $90 \%$ y a los 5 años es de $62 \%$. $^{2,4}$

\section{Fisiopatología y etiología}

El síndrome de Churg Strauss (SCS) tiene una fisiopatología compleja, en la cual contribuyen factores genéticos y agentes extrínsecos; también, diferentes tipos de células, como células T, eosinófilos, células B y células residentes participantes en la reacción inflamatoria y la lesión tisular.

Determinantes genéticos: GEPA es una enfermedad asociada a HLA: 2 estudios independientes realizados en un cohorte Italiano y Alemán publicaron resultados similares, demostrando que los alelos HLA-DRB1_04 y _07 y el gen relacionado HLADRB4 confieren una susceptibilidad aumentada para síndrome de Churg Strauss, mientras que el HLA-DRB1_13 y el gen relacionado HLA-DRB3 son protectores. El hallazgo de un repertorio restringido de HLA de clase II sugiere que las células T CD4 están involucrados en la patogénesis de SCS y que la enfermedad puede ser mediada por antígenos. La IL10 es una citoquina Th2, así un aumento de su producción determinado genéticamente puede ser relevante para enfermedades mediadas por Th2 como el SCS.

Los alérgenos inhalados, las infecciones, vacunas y otras drogas han sido descritos como desencadenantes potenciales de SCS. Los antagonistas de los receptores de leucotrienos (ARLT) utilizados en el asma han sido frecuentemente implicados. Un análisis reciente de los casos de SCS en sospecha de ser inducidos por drogas, registrados en la base de datos del sistema de eventos adversos de la FDA ha demostrado que los ARLT fueron medicamentos sospechosos en la mayoría de los casos confirmados.

El rol de la alergia en el SCS se da por sentado, especialmente en la fase prodrómica de la enfermedad que se caracteriza por manifestaciones alérgicas típicas como asma, sinusitis y polipósis nasal; además, los niveles séricos de IgE están elevados en aproximadamente el $90 \%$ con SCS. A pesar de eso el asma en el SCS tiene un curso no convencional pues tiene un inicio en la adultez y mejora paradójicamente cuando los síntomas de vasculitis se desarrollan. 
Rol de las células $T$ : las células $\mathrm{T}$ son abundantes en las lesiones del SCS, y en algunas de ellas (neuropatía periférica) representan las células inflamatorias predominantes. El SCS ha sido tradicionalmente considerado como una enfermedad mediada por Th2, principalmente debido a su componente alérgico prominente, de hecho numerosos T CD4 que expresan marcadores Th2 infiltran lesiones en el SCS y las líneas de células T CD4 periféricas producen grandes cantidades de citoquinas $\mathrm{Th} 2$, sin embargo las respuestas $\mathrm{Th} 2$ no explican totalmente la patología del SCS, especialmente la ocurrencia de granulomas y de vasculitis.

La línea de las células T CD4 de pacientes con SCS también secretan cantidades significativas de interferón-g (IFNg) una potente citoquina Th1 involucrada en la formación de vasculitis y granulomas. Aunque las respuestas Th2 probablemente predominan en el SCS la inmunidad por Th1 y Th17 pueden también contribuir en la patogénesis de la enfermedad.

Anormalidades de eosinófilos: los eosinófilos son ciertamente mediadores claves del daño tisular en el SCS, sin embargo no deben ser únicamente considerados como células efectoras debido a que también pueden instruir otros tipos de células inflamatorias e influir en sus propiedades funcionales. Un estudio reciente demostró que los niveles de IL25 estaban aumentados en pacientes con SCS, la IL 25 estimula a los linfocitos periféricos para que produzcan citoquinas Th2 típicas como: IL4, IL5 e IL13, que a su vez producen eosinofilia. Una estimulación orquestrada por citoquinas Th2 y quimiocinas estimulan a los eosinófilos para que se acumulen en la circulación e infiltren en los tejidos blancos.

Anticuerpos citoplasmáticos antineurófilos, producción de IgG y células B: los autoanticuerpos como los anticuerpos citoplasmáticos antineutrófilos (ANCA), son detectables entre el 30 al $40 \%$ de los pacientes. Aunque su patogenicidad en el SCS sigue siendo incierta, su fuerte asociación con las manifestaciones de vasculitis en la enfermedad parece coherente con esta hipótesis. El rol de las células B en el SCS ha sido recientemente revisado. La depleción de las células B con rituximab se ha convertido en un tratamiento bien establecido para las vasculitis asociadas a ANCA y durante los últimos años numerosos reportes de casos de SCS refractario han mostrado una respuesta positiva al rituximab, interesantemente el rituximab induce una reducción significativa de los conteos de los eosinófilos. ${ }^{(5)}$

\section{Cuadro clínico}

Se han descrito tres fases de evolución de la enfermedad parcialmente superpuestas. Una primera fase prodrómica caracterizada por manifestaciones atópicas como asma, rinitis alérgica, sinusitis y pólipos nasales. El asma se caracteriza por ser de aparición tardía (en el adulto), y puede llegar a ser refractaria al manejo convencional. Puede preceder la aparición de la vasculitis entre tres y ocho años. La segunda fase se caracteriza por eosinofilia periférica, y pueden ocurrir infiltrados eosinofílicos en pulmones y tracto gastrointestinal. La eosinofilia usualmente es mayor del $10 \%$ del conteo leucocitario o mayor de 1500/mm3. En esta fase se presentan síntomas constitucionales como fiebre y pérdida de peso.

Durante la tercera fase de la enfermedad ocurren manifestaciones vasculíticas. Además de los síntomas constitucionales también hay manifestaciones osteomusculares como mialgias, artralgias y artritis. Los reactantes de fase aguda se encuentran elevados por lo general. Se han descrito casos de compromiso limitado a un solo órgano.

El compromiso neurológico es frecuente, siendo la neuropatía periférica mucho más prevalente que en las otras vasculitis de pequeños vasos asociadas con ANCA. La neuropatía periférica en el SCS ocurre en un 50\% a 75\% de los casos. Típicamente ocurre mononeuritis múltiple ocasionada por la lesión vasculítica de la vasa nervorum. Ocasionalmente la lesión neuropática periférica puede tener alguna mejoría con la terapia pero en general el daño suele ser permanente y causando importante morbilidad y discapacidad funcional. Aunque el compromiso del sistema nervioso central es menos frecuente, es la segunda causa de muerte en SCS y se presenta con infarto o hemorragia cerebral.

El compromiso gastrointestinal puede manifestarse con un abdomen agudo o dolor anginoso postprandial, colecistitis, hemorragia y perforación intestinal. La presencia de microaneurismas en circulación esplácnica con cuadros de isquemia mesentérica ha sido descrita; en una serie de 96 pacientes con SCS se encontraron microaneurismas en 9 de 26 pacientes a quienes se les realizó angiografía celiaco-mesentérica y/o renal. Las manifestaciones cutáneas se presentan en aproximadamente el 50\% 
de los pacientes, siendo las más frecuentes, la púrpura palpable y las petequias en las extremidades inferiores, y los nódulos y pápulas en superficies extensoras de las articulaciones, más comúnmente en codos.

Otras lesiones descritas son maculopápulas eritematosas tipo eritema multiforme, úlceras, livedo reticularis y edema facial. La presencia en biopsia de piel de granuloma necrosante extravascular (granuloma Churg-Strauss) se correlaciona clínicamente con lesiones tipo pápulas y nódulos, mientras que lesiones tipo petequias y púrpura se correlacionan con vasculitis leucocitoclástica en la biopsia. La enfermedad cardiaca es la principal causa de muerte y es responsable del $50 \%$ de los fallecimientos. Puede ocurrir falla cardiaca por miocarditis y asociarse con pericarditis e hipertensión. Con menor frecuencia se ha reportado isquemia coronaria, cardiomiopatía restrictiva y arritmias. En pulmón puede haber infiltrados pulmonares, derrame pleural, nódulos que rara vez forman cavernas, y hemorragia pulmonar secundaria a capilaritis. Las alteraciones renales son inusuales y suelen responder rápidamente al manejo. ${ }^{6}$

Comarmond et al realizan un estudio retrospectivo de pacientes con SCS del grupo del estudio cohorte francés de vasculitis, que cumplían con los criterios del Colegio Americano de Reumatología y/o con las definiciones de Chapel Hill, con el objetivo de determinar las principales características de la enfermedad y compararlos de acuerdo a los diferentes estados ANCA y año de diagnóstico. Identificaron 383 pacientes diagnosticados entre 1957 y Junio del 2009 que fueron seguidos por un promedio de $66.8 \pm 62.5$ meses y la edad promedio fue de 50.3 \pm 15.7 años.

Las manifestaciones más comunes de GEPA al diagnóstico incluyeron: pérdida de peso $(49.3 \%)$, mononeuritis multiples (46\%), sinusitis/poliposis no erosiva $(41.8 \%)$, lesiones cutáneas $(39.7 \%)$, e infiltrados pulmonares $(38.6 \%)$. Se reportó derrame pleural en 34 (8.9\%). la afectación cardiaca, gastrointestinal y renal, se encontró en $27.4 \%$, $23.2 \%$ y $21.7 \%$ de los pacientes respectivamente. El $50 \%$ de los pacientes tenían un síndrome inflamatorio y el promedio de conteo de eosinófilos fue de $7569 \pm 6428 / \mathrm{mm}^{3}$.

Del total de pacientes en 35 no se pudo determinar el estado ANCA, de los restantes 348 pacientes, $108(31 \%)$ fueron ANCA positivo
Características de los pacientes al diagnóstico de acuerdo al estado ANCA, el año de diagnóstico y sexo: el estudio demostró que los pacientes ANCA positivos tenían una frecuencia significativamente mayor de manifestaciones en oídos, nariz y garganta, afectación de nervios periféricos, y enfermedad renal que los pacientes ANCA negativo.

De lo contrario los pacientes ANCA negativos tienen una frecuencia significativamente mayor de cardiomiopatía. Entre los pacientes en los que las biopsias apoyaban el diagnóstico del SCS, las características histológicas de vasculitis fueron más frecuentes en los pacientes ANCA positivo (77.4\% vs $48.8 \%$ en pacientes ANCA negativos), mientras que los infiltrados granulomatosos y eosinofílicos, se vieron en $42.1 \%$ y $63.2 \%$, respectivamente, de los pacientes ANCA positivos, vs $29 \%$ y $69.7 \%$, respectivamente, de los pacientes ANCA negativos.

Los pacientes diagnosticados durante o previo a 1996, comparados con aquellos diagnosticados después de 1996, fueron más jóvenes al diagnósticos y tenían con mayor frecuencia síntomas constitucionales, asma, mononeurítis múltiple, cardiomiopatía y enfermedad renal, comparados con los pacientes masculinos, las pacientes femeninos con GEPA fueron significativamente más jóvenes al diagnóstico, tuvieron un duración más larga antes del diagnóstico de GEPA y tuvieron menos anormalidades renales. ${ }^{7}$

\section{Clasificacion y diagnóstico}

Para su clasificación el American College of Rheumatology (1990) publicó los siguientes criterios diagnósticos contando con al menos cuatro de los siguientes seis:

1. Asma: historia de dificultad respiratoria o sibilancias espiratorias difusas.

2. Eosinofilia: eosinofilia $>10 \%$ en conteo diferencial de glóbulos blancos.

3. Mono o polineuropatía: desarrollo de mononeuropatía múltiple, o polineuropatía (distribución en guante o en bota) atribuible a vasculitis sistémica. 
4. Infiltrados pulmonares migratorios: infiltrados pulmonares migratorios o transitorios (no incluyendo infiltrados fijos). Atribuibles a vasculitis.

5. Anormalidades de los senos paranasales: historia de dolor agudo o crónico de los senos paranasales u opacificación radiográfica de los senos paranasales.

6. Eosinófilos extravasculares: biopsia incluyendo arterias, arteriolas o vénulas mostrando acúmulos de eosinófilos en áreas extravasculares.

Los criterios del Colegio Americano de Reumatología solamente pueden utilizarse para la clasificación del síndrome de vasculitis como el SCS, con una sensibilidad de $85 \%$ y una especificidad de $99.7 \%$ cuando al menos 4 de los criterios están presentes, sin embargo no puede utilizarse para el diagnóstico de SCS. Las definiciones de la conferencia de consenso de Chapel Hill eran principalmente descriptivas pero no son adecuadas para el diagnóstico ni la clasificación del SCS. A pesar de eso, debido a la falta de herramientas diagnosticas validas, los criterios de CAR y las definiciones de la $\mathrm{CCCH}$ frecuentemente se les dan erróneamente significancia diagnóstica. La evolución clásica de SCS en 3 fases diferentes provee, en algunos casos, una herramienta diagnostica. La fase prodrómica, la cual puede persistir por muchos años, es caracterizada por asma alérgica, así como rinitis y pólipos nasales que tienden a recurrir despúes de la cirugía. La segunda fase se caracteriza por eosinofilia periférica e infiltrados eosinofilicos tisulares que pueden afectar el tracto respiratorio, gastrointestinal y el miocardio. En la tercera fase predominan los síntomas constitucionales y las manifestaciones de afectación de órganos por vasculitis.

La secuencia de fases no siempre es bien definida pueden superponerse o algunas de ellas pueden estar no presentes del todo, por lo que pueden ser útiles para el médico solo cuando se encuentran en el orden típico, entre los exámenes de laboratorios que apoyan el diagnostico de SC, la eosinofilia sanguínea $(>$ de $10 \%$ o $>$ de 1500 celulas/ $\mu$ l) es extremadamente frecuente. Los niveles elevados de PCR y VES son comunes al igual que la anemia. Los niveles séricos totales de IgE están elevados en muchos casos. También se puede encontrar niveles elevados de proteína eosinofílica catiónica y con menor frecuencia factor reumatoide.
Zwerina et al. Brindan una contribución efectiva al diagnóstico de SCS en su fase activa, estos autores demostraron que la eotaxina-3 (una citoquina con efectos de quimotaxis, transmigración celular y movilización del flujo de $\mathrm{Ca}^{+2}$ sobre los eosinófilos) estaba significativamente elevada en el SCS activo, y que claramente diferencia el SCS activo del inactivo. Algunas características genéticas pueden potencialmente de ayuda para el diagnóstico de SCS: el HLA-DRB4, es un factor de riesgo genético bien demostrado para el desarrollo de SCS y probablemente esté ligado al desarrollo de las manifestaciones de vasculitis de la enfermedad, al contrario el haplotipo IL-10.2 se asocia significamente con SCS ANCA negativo.

Sin embargo estos polimorfismos genéticos no han sido evaluados en otros grupos de vasculitis, por lo tanto su rol en el diagnóstico diferencial es incierto. $(8,9)$

\section{Tratamiento}

El pronóstico del SCS ha mejorado dramáticamente desde la introducción de los corticoesteroides, y estos siguen siendo la primera línea de terapia de la GEPA. Aunque la piedra angular del tratamiento son los corticoesteroides, la enfermedad severa es tratada de manera similar a otras vasculitis asociadas a ANCA, con otros agentes inmunosupresores como ciclofosfamida. Sin embargo el manejo sigue siendo un reto, con estudios clínicos limitados. El tratamiento debe adaptarse a la severidad de la enfermedad, el manejo de pacientes con enfermedad multisistémica que pone en riesgo la vida, debe llevarse a cabo en conjunto con un médico con experiencia y pericia. 
Figura 1. Diagnostico diferencial del scs con base a hallazgos clínicos de laboratorio, e histologicos

\begin{tabular}{|c|c|c|c|c|}
\hline Diagnostic features & css & HES & AASV & CTD \\
\hline Constitutional symptoms & +++ & + & +++ & +++ \\
\hline \multicolumn{5}{|l|}{ Ear, nose, throat } \\
\hline Sinusitis/rhinitis & +++ & - & $+++(W G)$ & - \\
\hline Nasal polyposis & +++ & +- & +- & - \\
\hline \multicolumn{5}{|l|}{ Lung } \\
\hline Asthma & +++ & +- & +- & - \\
\hline Non-fixed infiltrates & +++ & +++ & + & - \\
\hline Nodules & +- & - & ++ & - \\
\hline Eosinophilia in BAL & +++ & +++ & + & +- \\
\hline Alveolar haemorrhage & + & - & ++ & - \\
\hline Pleural effusion & + & +- & + & ++ \\
\hline \multicolumn{5}{|l|}{ Cardiovascular } \\
\hline Myocarditis & + & ++ & - & - \\
\hline Pericarditis & + & +- & +- & + \\
\hline Coronary vasculitis & ++ & - & + & - \\
\hline \multicolumn{5}{|l|}{ Nervous system } \\
\hline Central & + & + & + & + \\
\hline Peripheral & ++ & +- & ++ & +- \\
\hline \multicolumn{5}{|l|}{ Gastrointestinal } \\
\hline Eosinophilic gastroenteritis & ++ & ++ & - & - \\
\hline Abdominal pain & ++ & - & + & + \\
\hline \multicolumn{5}{|l|}{ Kidney } \\
\hline GN & ++ & - & +++ & ++ \\
\hline RPGN & +- & - & +++ & ++ \\
\hline Interstitial nephritis & + & + & + & + \\
\hline \multicolumn{5}{|l|}{ Skin } \\
\hline Rash & + & - & + & ++ \\
\hline Purpura & ++ & - & ++ & + \\
\hline \multicolumn{5}{|l|}{ Laboratory } \\
\hline Eosinophilia & +++ & +++ & + & +- \\
\hline ESR & +++ & - & +++ & +++ \\
\hline $\lg E$ & +++ & + & + & - \\
\hline pANCA & ++ & - & $+++(\mathrm{MPA})$ & - \\
\hline CANCA & + & - & $+++(\mathrm{WG})$ & - \\
\hline \multicolumn{5}{|l|}{ Histology } \\
\hline Necrotizing vasculitis & ++ & - & ++ & +- \\
\hline Eosinophil tissue infiltration & ++ & ++ & - & - \\
\hline Granuloma & ++ & - & ++ & - \\
\hline
\end{tabular}

Fuente: Rheumatology, 50(10), pp.1737-1738. La frecuencia de las características enlistadas se gradúa desde +++ (muy frecuente) a (usualmente ausente). HES: síndrome hipereosinofílico; AASV: vasculitis sistémicas asociadas a ANCA; CTD: enfermedades del tejido conectivo; BAL: lavado bronquioalveolar.

La escala de 5 factores clasifican a los pacientes en alto y bajo riesgo. Aquellos con una escala de los 5 factores $\geq 1$ requieren una terapia inmunosupresora a una escala similar a la requerida en la granulomatosis severa con poliangeítis (GPA). Aunque la remisión se logra en más de un $80 \%$ de los pacientes, la GEPA es comúnmente una enfermedad reincidente (10 a 43\%).

\section{Escala de 5 factores de indicadores de mal pronóstico en poliarteritis nodosa y GEPA}

Esta escala permite la predicción de pronóstico de pacientes con GEPA. La presencia de alguno de estos 5 factores contribuye a la mortalidad y al resultado final:

1. Proteinuria $>1 \mathrm{~g} / 24$ horas

2. Creatinina sérica $>1.58 \mathrm{mg} / \mathrm{dl}(>140$ micromol/L)

3. Afectación del tracto gastrointestinal.

4. Miocardiopatía

5. Afectación del SNC.

Cuando ninguno de estos 5 factores, la mortalidad en 5 años es de $11.9 \%$, la mortalidad incrementa a medida que incrementan estos 5 factores, con un aumento en la mortalidad a $49.95 \%$ cuando al menos 2 factores están presentes.

Escala de 5 factores $<1$ : se da una dosis alta de prednisolona, con o sin metilprednisolona pulsada intravenosa. Una vez que la enfermedad remite, basado en los síntomas y marcadores de inflamación, los corticoesteroides pueden disminuirse lentamente por varios meses. Los pacientes pueden que requieran mantenimiento con dosis bajas de prednisolona( $<$ de $10 \mathrm{mg} / \mathrm{dia}$ ) a largo plazo, esto puede requirse para controlar los síntomas del asma. Si la dosis de corticoesteroides no puede disminuirse a menos de $10 \mathrm{mg}$ por dia de prednisolona y el asma no es la causa, la introducción de otro agente como metrotexate o azatriopina es razonable. En algunos países utilizan ciclofosfamida.

Escala de 5 factores $\geq 1$ : están indicados ciclofosfamidas más corticoesteroides en presencia de factores de pobre pronóstico. Pueden darse intravenosos u orales. La vía intravenosa es particularmente recomendada en mujeres jóvenes con deseos de preservar la fertilidad, y en aquellos pacientes en los que el cumplimiento del tratamiento puede ser un problema. Esto es debido a que la terapia intravenosa permite una dosis menor, aunque puede caerse con un rango mayor de reincidencia.

Típicamente se administra mesna para evitar complicaciones vesicales por la ciclofosfamida intravenosa y la dosis oral se ingieren en la mañana con cantidades copiosas de líquidos para prevenir toxicidad de la vejiga, deben monitorearse el conteo de 
células sanguíneas semanalmente. La inmunogloblulina intravenosa no se utiliza GEPA en EEUU.

Mientras que las ciclofosfamida ha sido tradicionalmente el estándar de tratamiento para las vasculitis severas asociadas a ANCA, el rituximab (anticuerpo antiCD20) ha demostrado efectividad. Tiene ahora licencia para su uso en GPA en EEUU y aunque los grandes estudios solo incluyen pacientes con GPA y MPA se ha reportado su eficacia en GEPA en reportes de casos además, el estándar clínico es tratar la GEPA con manifestaciones vasculiticas severas de la mismsa manera que otras vasculitis asociadas a ANCA y esto se ve reflejado en las guías del 2014 de la Sociedad Britanica de Reumatología para el manejo de adultos con vasculitis asociadas con ANCA. El rituximab es el tratamiento de referencia cuando hay razones para evitar la ciclofosfamida (preservar la fertilidad)

Terapia adyuvantes: aunque en la práctica puede variar, los pacientes que toman más de $20 \mathrm{mg} / \mathrm{d}$ de prednisolona o agentes economizadores de corticoesteroides, reciben profilaxis contra Pneumocystis jirovecii. Comúnmente se realiza con trimetoprin sulfametoxazol. En pacientes con alergias a las sulfas, las alternativas incluyen dapsona, atovacuona o pentamidina inhalada. Los pacientes que consumen corticoesteroides a largo plazo deben también recibir suplementación con calcio y vitamina D.

Existe interés en tratar de inducir una menopausia química temporal en mujeres jóvenes que reciben ciclofosfamida en un intento por preservar la fertilidad. Esto se realiza con manipulación hormonal con el análogo de GnRH, leuprolelin. En hombres se recomienda un banco de esperma antes de iniciar terapia con ciclofosfamida. En circunstancias en que la enfermedad severa y pone en riesgo la vida, un intercambio de plasma puede utilizarse como adyuvante a los costicoesteroides y a la ciclofosfamida.

\section{Fármacos emergentes:}

- Interferón alfa: actúa en las células T, influyendo en la producción de citoquinas.

- Anti-interleucina (IL)-5: se encuentra en niveles elevados de IL5 en la GEPA activa. En EEUU el agente anti-IL5, mepolixumab está aprobado por la FDA para el tratamiento del asma eosinofilica. Un estudio para determinar la eficacia de mepolixumab en GEPA, está en proceso.

- Omalixumab: anticuerpo monoclonal anti-IgE humana. Inhibe la unión de la $\mathrm{IgE}$ con los receptores de $\mathrm{IgE}$ y se une a la IgE libre en el plasma, muchos casos reportados de GEPA han ocurrido durante la terapia con omalixumab, aunque se necesitan más estudios para determinar eficacia y seguridad. ${ }^{10}$

\section{CONCLUSIONES}

El síndrome de Churg Strauss entra dentro del grupo de enfermedades llamadas vasculitis de pequeños vasos, se describe en la bibliografía 3 fases evolutivas de la enfermedad que no siempre ocurren en un orden específico y que pueden solaparse, la fisiopatología que explica la enfermedad es compleja contribuyendo factores genéticos e inmunológicos, con algunos agentes ambientales determinados como desencadenantes.

Un dato característico del cuadro clínico es la presencia de asma de aparición tardía, en el caso presentado el diagnóstico se realizó aproximadamente a los 28años. Los criterios del American College of Rheumatology describen 6 criterios en los que se deben cumplir 4 para la clasificación de Síndrome de Churg Strauss, sin embargo se menciona en la bibliografía que no deben utilizarse como diagnósticos. Los corticosteroides son descritos como la piedra angular del tratamiento, y el manejo varía de acuerdo a la severidad de la enfermedad añadiéndose generalmente ciclofosfamida cuando hay presentes factores de pobre pronóstico. El manejo es un reto, y aunque en la mayoría de pacientes se logra la remisión de la enfermedad ésta es comúnmente una patología reincidente.

\section{BIBLIOGRAFÍA}

1. Groh M Pagnoux C Guillevin L. Eosinophilic granulomatosis with polyangiitis (formerly Churg-Strauss syndrome): where are we now?. Eur Respir J. 2015; 46: 1255-1258 
2. Farid-Moayer M. Churg-Strauss Syndrome. Medscape. 2015; http://emedicine.medscape.com/article/333492-overview.

3. Sánchez A González M. Poliangeítis granulomatosa con eosinofilia. Revisión y reporte de caso de una paciente con eosinofilia, mononeuropatía de miembro inferior, rinosinusitis y asma. Revista Colombiana de Reumatología. 2015; 22(3): 180-185.

4. Staley R. Churg-Strauss Disease Clinical Presentation. Medscape. 2104; http://emedicine.medscape.com/article/1178795-clinical

5. Vaglio A Moosig F Zwerina J. ChurgStrauss syndrome: update on pathophysiology and treatment. Curr Opin Rheumatol. 2012 Jan; 24(1): 24-30

6. Restrepo M González L Vásquez G Londoño D Correa L Ramírez L. Síndrome de Churg-Strauss: a propósito de un caso

\section{CONFLICTO DE INTERÉS Y/O AGRADE- CIMIENTOS}

Los autores declaran que no existió ningún conflicto de interés en el presente reporte. con manifestaciones poco usuales. Revista Colombiana de Reumatología. 2009; 16 (1): 112-119.

7. Comarmond C Pagnoux $\mathrm{C}$ Khellaf $\mathrm{M}$ et al. Eosinophilic granulomatosis with polyangiitis (Churg-Strauss): clinical characteristics and long-term followup of the 383 patients enrolled in the French Vasculitis Study Group cohort. Arthritis Rheum. 2013; 65(1): 270-281.

8. Buzio C Oliva E. Diagnosis of Churg Strauss syndrome: eotaxin-3 makes it easier. Rheumatology 2011; 50: 1737 1738.

9. Terán L Ledesma Y Krengel S Lezcano D. Eotaxinas en asma bronquial y poliposis nasal. Gac Méd Méx. 2006; 142 (2): 139144

10. Keogh K. Eosinophilic granulomatosis with polyangiitis (Churg-Strauss sindrome). BMJ Best Practice. 2016; http://bestpractice.bmj.com/best-practice/mybp/monograph-pdf/942.pdf 